\title{
Do Comorbidities Influence the Treatment of Myocardial Infarction?
}

A cute myocardial infarction (AMI) is the leading cause of death in the United States, with roughly 225,000 deaths resulting from 900,000 AMIs each year. ${ }^{1}$ Despite numerous large, randomized trials that show a decrease in AMI mortality with therapies such as thrombolytics and aspirin, there is evidence that these therapies are underutilized in this country. Thrombolysis has been shown to decrease short-term mortality of AMI by about $18 \%,{ }^{2}$ with a risk of cerebral hemorrhage of less than $0.5 \% .^{3} \mathrm{Al}-$ though studies have shown that one-third of patients were eligible to receive intravenous thrombolysis at the time of presentation with AMI, only about half of these patients actually received this therapy. ${ }^{4}$ Similarly, aspirin given immediately and for 30 days after an AMI decreased mortality by $23 \%$ within the first 5 weeks. ${ }^{5}$ However, only $84 \%$ of eligible patients received aspirin. ${ }^{6}$ Although prior research has demonstrated that use of these drugs is less than expected, few have examined the possible explanations for this occurrence.

In the article that appears in this issue, McLaughlin et al. ${ }^{7}$ attempt to assess the role of physicians' knowledge of underlying medical conditions, other than those known to represent contraindications for therapy, in the decision to administer either thrombolysis or aspirin at the time of a patient's initial presentation with AMI. In their retrospective analysis, the authors applied a comorbidity score to 2,409 patients at 37 Minnesota Hospitals who presented with AMI. Interestingly, they found that patients with severe comorbidities who met eligibility criteria for thrombolysis and aspirin were half as likely to receive those therapies compared with patients without comorbidities. Although the results are provocative, issues of study design must be addressed to place the conclusions in a proper perspective.

Missing information is a common problem in retrospective studies. Because information on critical study variables relies exclusively on the availability of previously collected data, misclassification of absent data as "not present" could bias the results. If missing data are evenly distributed between those patients who did and did not receive thrombolysis or aspirin, then the results may be biased toward the null hypothesis, and the observed disparities would therefore be an underestimate of the true differences in treatment between the two groups. However, if information on comorbidities was more likely to be recorded in those patients not receiving thrombolysis or aspirin, even if unrelated to the decision to not use these therapies, a false association between comorbidities and lack of treatment would result.
The fact that comorbidities were also related to aspirin use in McLaughlin et al.'s study, an association that is difficult to understand given the known effects and safety profile of aspirin, suggests that bias as described above may explain some of the findings in this study. ${ }^{7}$ While legitimate concerns may exist over administering thrombolytics to patients with certain comorbidities, it is difficult to understand these apprehensions with aspirin. In ISIS-2, major bleeding complications occurred in only $0.4 \%$ in both aspirin- and placebo-treated patients. ${ }^{5}$ In addition, true contraindications to aspirin, such as known hypersensitivity, are infrequent occurrences. At a cost of approximately one cent per dose, aspirin makes economic sense as well; it is estimated that it would cost only $\$ 13$ for each premature death avoided by using aspirin. ${ }^{8}$

Also lacking are important details concerning those patients who were denied the therapies under question. For example, since the leading cause of comorbidity was mental impairment, it would be useful to know whether the inability to obtain informed consent was a major factor in the decreased likelihood of receiving thrombolysis. This problem would obviously not apply to aspirin therapy. It is also possible that patients with some of the comorbidities mentioned may have been more likely to be on aspirin as outpatients, thereby influencing the physician's decision to administer a second dose at the time of presentation. Lastly, we are not told what percentage, if any, of those patients in whom primary thrombolytic therapy was withheld were treated with primary angioplasty. This may be pertinent information, since a patient in whom a comorbidity may dissuade a physician from giving potentially harmful therapies like thrombolytics may be more apt to be treated with primary angioplasty instead.

Despite these limitations and the inability of this study to examine directly the reason for withholding therapy, McLaughlin et al. raise an important hypothesis: the apparent underuse of potentially lifesaving therapies may reflect, in part, a conscious decision by physicians to withhold these therapies in patients whom they feel are unlikely to benefit long-term. Underuse of drug therapy may still reflect gaps in physicians' knowledge or lack of acceptance of treatment guidelines, although one recent study has shown that clinical trials do affect physicians' use of aspirin and calcium channel blockers in the treatment of AMI. ${ }^{9}$ It seems likely that the multitude of studies confirming the efficacy of thrombolysis would increase use of this drug. Nonetheless, efforts at improving physi- 
cians' knowledge and use of proven therapies remain important issues. Also, whether a decision to withhold therapies in patients with a severe comorbidity is appropriate is another important and controversial issue. ${ }^{10,11}$

At a time of rapid growth of clinical pathways, treatment guidelines, and ever-larger clinical trials, medicine remains an art, where existing knowledge gleaned from studies on populations must be applied to the individual. Within the broad categories of "eligible" and "ineligible" for any given treatment are many gradations, especially in the case of potentially harmful therapies. McLaughlin et al. raise one possibility that "explains" some of the underuse of two such therapies. ${ }^{5}$ Future, preferably prospective, studies should address this extremely important issue.Rod Passman, MD, and STePhen Kimmel, MD, MS, Department of Medicine, University of Pennsylvania School of Medicine, Philadelphia.

\section{REFERENCES}

1. ACC/AHA Guidelines for the Management of Patients With Acute Myocardial Infarction. J Am Coll Cardiol. 1996;28:1328-1419.

2. Fibrinolytic Therapy Trialists' (FTT) Collaborative Group. Indications for fibrinolytic therapy in suspected acute myocardial infarction: collaborative overview of early mortality and major morbidity results from all randomised trials of more than 1000 patients. Lancet. 1994;343:311-22.

3. Ridker PM, Marder VJ, Hennekens CH. Large-scale trials of thrombolytic therapy for acute myocardial infarction: GISSI-2, ISIS-3, and GUSTO-1. Ann Intern Med. 1993;119:530-2.

4. Muller DW, Topol EJ. Selection of patients with acute myocardial infarction for thrombolytic therapy. Ann Intern Med. 1990;113: 949-60.

5. ISIS-2 Collaborative Group. Randomised trial of intravenous streptokinase, oral aspirin, both, or neither among 17,187 cases of suspected acute myocardial infarction: ISIS-2. Lancet. 1988; August 13:349-60.

6. Rogers WJ, Bowlby LJ, Chandra NC, et al. Treatment of myocardial infarction in the United States (1990 to 1993). Circulation. 1994;90:2103-14.

7. McLaughlin IJ, Soumerai SB, Willison DJ, et al. The effect of comorbidity on use of thrombolysis or aspirin in acute myocardial infarction patients eligible for treatment. $J$ Gen Intern Med. 1997;12:1-6.

8. Hennekens $\mathrm{CH}$, Jonas MA, Buring JE. The benefits of aspirin in acute myocardial infarction. Arch Intern Med. 1994;154:37-9.

9. Lamas GA, Pfeffer MA, Hamm P, Werthheimer J, Rouleau J, Braunwald E, For the SAVE Investigators. Do the results of randomized clinical trials of cardiovascular drugs influence medical practice? N Engl J Med. 1992;327:241-7.

10. Schneiderman LJ, Jecker NS, Jonsen AR. Medical futility: response to critiques. Ann Intern Med. 1996;125:669-74.

11. Caplan AL. Odds and ends: trust and the debate over medical futility. Ann Intern Med. 1996;125:688-9. 